Para enlazar con este artículo / To link to this article:

http://dx.doi.org/10.6035/MonTI.2021.13.01

Para citar este artículo / To cite this article:

Calzada Pérez, María \& Sara Laviosa. (2021) "Twenty-five years on: Time to pause for a new agenda for CTIS." In: Calzada, María \& Sara Laviosa (eds.) 2021. Reflexión crítica en los estudios de traducción basados en corpus / CTS spring-cleaning: A critical reflection. MonTI 13, pp. 7-32.

\title{
TWENTY-FIVE YEARS ON: TIME TO PAUSE FOR A NEW AGENDA FOR CTIS ${ }^{1}$
}

\author{
MARÍA CALZADA PÉREZ \\ calzada@uji.es \\ Universitat Jaume I \\ SARA LAVIOSA \\ sara.laviosa@uniba.it \\ University of Bari Aldo Moro
}

\begin{abstract}
The introduction of corpora in descriptive and applied translation and interpreting studies goes back to the 1990s, when the corpus linguistic approach was making considerable progress in descriptive and applied language studies. Twenty-five years on, Corpus-Based Translation and Interpreting Studies (CTIS) is a well-established field of interdisciplinary research worldwide. Its growth goes hand in hand with technological advancements, which make it possible to design, create and share monolingual and multilingual spoken, written and multimodal corpora as resources for theoretical, descriptive and applied research in both translation and interpreting studies. We believe this is the right time to pause and reflect on the achievements and criticalities of this variegated area of scholarship and practice in order to look to the future with renewed confidence and awareness of the challenges that lie ahead.
\end{abstract}

1. This article was partly carried out within the research project Representaciones originales, traducidas e interpretadas de la(s) crisis de refugiados: triangulación metodológica desde el análisis del discurso basado en corpus (RE-CRI), financially supported by Ministerio de Ciencia e Innovación (PID2019-108866RB-I00). 
Keywords: Corpus-based Translation Studies; Corpus-based Interpreting Studies; Corpus Linguistics; Corpus-assisted Discourse Analysis; Contrastive Linguistics.

\section{Resumen}

La introducción de los corpus en los estudios descriptivos y aplicados de traducción e interpretación se remonta a la década de 1990. Han pasado ya (más de) 25 años y los estudios de traducción e interpretación basados en corpus (CTIS) son un campo de investigación interdisciplinar bien establecido en todo el mundo. Su crecimiento va de la mano de los avances tecnológicos, que permiten diseñar, crear y compartir corpus monolingües y multilingües (orales, escritos y multimodales) como recursos para la investigación teórica, descriptiva y aplicada en los estudios de traducción e interpretación. Creemos que es el momento oportuno para hacer una pausa y reflexionar sobre los logros y las carencias de este variado ámbito de la erudición y la práctica, con el fin de mirar al futuro con renovada confianza y conciencia de los retos que nos esperan.

Palabras clave: Estudios de traducción basados en corpus; Estudios de interpretación basados en corpus; Lingüística de corpus; Análisis del Discurso Asistido por Corpus; Lingüística contrastiva.

\section{The origins of Corpus-based Translation and Interpreting Studies}

Corpus-based Translation Studies (CTS) denotes an area of research that adopts and develops the methodologies of Corpus Linguistics (CL) to analyse translation practices for theoretical, descriptive and applied purposes. CL is an approach to language studies, which is based on the analysis of corpora, i.e. collections of authentic texts held in electronic form and assembled according to specific design criteria. In this article, we trace the development of CTS from its advent in the 1990s to the present day, highlighting its main achievements in various ambits of enquiry, with a view to providing the background to the collection of papers selected for this volume of MonTI.

After Mona Baker's (1993) seminal paper, where a research agenda for the corpus-based approach to translation studies was outlined, the first collection of papers devoted to this research area was published in 1998 in a special issue of Meta entitled L'Approchee Basée sur le Corpus/The CorpusBased Approach, guest edited by Sara Laviosa. The papers commissioned for this issue were grouped under two main headings: "Theoretical Research" 
and "Empirical and Pedagogical Studies." The first group outlines the scope, object of study, and methodology of the emergent corpus-based approach. The second group consists of empirical and pedagogical studies of the product and process of translation. The concluding paper by Maria Tymoczko draws on the insights provided by these essays and points out that investigating translation by means of corpora serves "to address not simply questions of language and linguistics, but also questions of culture, ideology, and literary criticism" (1998: 653).

More specifically, CTS is viewed as having a central role within the whole discipline of Translation Studies, because (1) it is committed to integrate linguistic and cultural studies and explore their interrelationship, (2) it shows awareness of the effect of ideology on the theory, practice and pedagogy of translation, and (3) it aims to adapt modern technologies to enhance theory, empirical research and practice for the benefit of translator training and the work of the practising professional translator. Hence, Tymoczko (1998: 658) encourages a view of CTS that offers "the opportunity to reengage the theoretical and pragmatic branches of Translation Studies, branches which over and over again tend to disassociate, developing slippage and even gulfs." Indeed, these early corpus-based studies already illustrate some of the main lines of enquiry that, as envisaged by Tymoczko, would be developed in the years that followed. We now critically review each of these lines of enquiry in turn, with a view to showing how this scholarly research has enhanced our understanding of translation and translating up until now, and will continue to do so in future.

Three research domains can be identified in the late 1990s, each of them concerning issues and topics that fall within the realm of one of the three branches of the discipline as a whole, namely theoretical, descriptive and applied translation studies. As regards theory, the paper authored by Mona Baker (1998) deals with the rationale and motivation for investigating the product and process of translation through corpora expounding on the research agenda she had launched five years earlier. Baker discusses the need to develop a coherent corpus-based methodology for identifying the distinctive features of translational language. She argues that the aim of this research endeavour, which builds upon the studies of scholars working in the descriptive and target-oriented approach to Translation Studies, is not merely 
to unveil the nature of the 'third code' per se, but to understand the specific constraints, pressures and motivations that influence the act of translating intended as a mediated communicative event. These considerations seem to echo Tymoczko's appraisal of CTS as an approach whose "[m]odes of interrogation - as well as care in the encoding of metatextual information about translations and texts - allow researchers to move from text-based questions to context-based questions" (1998: 653).

In a similar vein, Miriam Shlesinger posits that just as translation is a communicative event shaped by its own goals, pressures and context of production, so too is interpreting. The term 'interpreting' is intended as "the production of oral output based on other-language input which may be either written (to be read) or unwritten (impromptu)" (1998: 486-487). Shlesinger proposes to extend Baker's notion of monolingual comparable corpora (consisting of a corpus of original texts in one language and a corpus of comparable texts translated into that same language) so as to comprise three collections of texts in the same language: interpreted speeches from a variety of source languages, original spoken texts produced in similar settings, and written translations of source oral texts delivered in analogous circumstances. This novel design would permit not only the study of interpreted texts as distinct instantiations of oral discourse, but also the identification of those regular patterns of language use that distinguish interpreting from written translation. Furthermore, Shlesinger proposes to adapt the traditional uniderectional parallel corpus design so as to include three sets of texts: source language texts, their interpreted versions, and their written translations. The particular advantage of this corpus type, she argues, is that it makes it possible to investigate language- and direction-specific features of the interpreted output along with their possible interaction with personal variable such as gender, extent of professional experience, or language background. On the basis of these considerations, Shlesinger envisages that the corpus-based study of interpreting will help scholars to continue to explore the common ground between interlingual written and spoken mediation as well as define what sets interpreting apart from translation (1998: 490-491).

With regard to parallel corpora, Kirsten Malmkjær (1998) explains the advantages of using these resources for contrastive and translation studies. For contrastive linguists, parallel corpora are valuable for investigating the 
differences and similarities in language use. For translation scholars, they are valuable for identifying translational norms. She then discusses two main problems connected with the use of parallel corpora for answering questions arising from within Translation Studies in particular. The first problem is that KWIC concordance lines do not always offer sufficient linguistic context to investigate features of whole texts. There exists, therefore, a risk that some aspects of translational behaviour may be revealed, while others may be overlooked. The second difficulty is related to the way parallel corpora are designed so as to include only one translation for each source text. This may hide an important aspect of the translational phenomenon, namely the differences existing between the various translations of the same original work. To remedy these shortcomings, Malmkjær suggests complementing norm-oriented studies, which require large amounts of text, with smaller and carefully constructed corpora which consist of one source text and as many translations of it as possible, so that in-depth investigations of entire texts can be performed. There are two advantages in combining these two different types of corpora. First, the findings would be richer, and second, they would be more rigorous, given that the larger corpus could be checked against the individual cases examined in the smaller corpus. Malmkjær argues that this methodology caters for the needs of both the contrastive linguist and the translation scholar, bringing them closer to one another in a relationship of mutual cooperation and encouraging synergies with bordering fields that, at any rate, have always been part of translation studies practices.

While Baker and Shlesinger explain the rationale for exploring translation and interpreting through corpora, and Malmkjær proposes to refine corpus-based methodology to address questions germane to contrastive and translation studies, Sandra Halverson (1998) discusses the issue of representativeness in the design of general purpose translation corpora and provides a coherent theoretical framework within which data and methodology form a coherent whole so as to ensure the comparability of empirical findings. To this end, Halverson proposes a prototypical conceptualisation of the object category as opposed to a classical one. In this approach, the target population is regarded as a prototype category whose centre is occupied - but only for the cultures of industrialised western countries - by professional translations, whereas the peripheral positions are filled in by clusters of 
different typologies, for instance those carried out by trainees or non-professional translators or those between one's own best language and another language. The relationship between the centre and the periphery within the prototype is not one of inclusion or exclusion of the elements belonging to the category, but of resemblance. Therefore, the boundaries of the different groups of translations are not impermeable. For the researcher this means that, in order to ensure representativeness, a sample corpus of the population of translated texts will have to be made up of an array of subcorpora enjoying different degrees of significance and all being regarded as legitimate objects of study. Given that prototypes are by definition culture-bound, corpus-based findings cannot be generalised beyond the specific target population that a given corpus represents.

Summing up, these early theoretical reflections focus on why, what and how we study translation and interpreting through corpora. We now move on to review the findings obtained from the empirical corpus studies of translation published in the special issue of Meta in 1998. Sara Laviosa's (1998b) investigation of English translated text is based on a subcorpus of the English Comparable Corpus (ECC). It comprises two collections of narrative prose in English: one is made up of translations from a variety of source languages (mainly romance languages), the other includes original English texts produced during a similar time span. The study reveals four patterns of lexical use in translated versus original texts: a relatively lower proportion of lexical words versus grammatical words, a relatively higher proportion of high-frequency versus low-frequency words, relatively greater repetition of the most frequent words, and less variety in the words most frequently used. Laviosa proposes to call these regularities in English translated text 'core patterns of lexical use' to indicate that, given their occurrence in both the newspaper (see Laviosa 1998a) and narrative prose subcorpora of ECC, they may prove typical of translational English in general.

Still within the quest for regular features of translational language, Linn Øverås investigates explicitation. She uses two subcorpora consisting of English and Norwegian translations of fiction, taken from the bi-directional English-Norwegian Parallel Corpus (ENPC). Øverås hypothesises a rise in the level of lexical and grammatical cohesion when translating from Norwegian into English and from English into Norwegian. The comparison 
of the distribution of explicitating and implicitating shifts reveals a general tendency to explicitate in both translational English and translational Norwegian, notwithstanding a lower level of explicitation in NorwegianEnglish translations. Øverås finds two types of increased cohesion, i.e. addition and specification. Addition involves the insertion of grammatical or lexical items not present in the source text. Specification involves the expansion or substitution of grammatical and lexical items present in the source text. Øverås considers several factors that can account for explicitation, for example, the stylistic preferences in the source and the target languages, the obligatory shifts resulting from target language grammatical rules or from culture-specific translation norms as well as the constraints inherent in translation as mediated communicative event. She also suggests linking explicitation with neutralization (the tendency to use common, unmarked collocations or similes rather than metaphors) because they both have the effect of achieving greater readability of the target text. In so doing, she implicitly highlights the interrelationship between different posited regular patterns of the 'third code'.

Moving on from the study of patterns to the study of shifts, Jeremy Munday reports on the preliminary findings of the analysis of Edith Grossman's translation, Seventeen Poisoned Englishmen, of a short story by Gabriel García Márquez, Diecisiete ingleses envenenados. Munday uses a variety of basic corpus linguistic analytical methods - word frequency lists, descriptive statistics and concordances - to explore texts inductively. Word frequency lists are first obtained for both source and target texts and then compared for identifying useful areas of investigation. Munday uses intercalated text, i.e. a text obtained by manually keying in the translated text between the lines of the source text. He then runs concordances of this intercalated text and uses them to carry out a contextualised comparative analysis of all the instances of selected lexical items in order to examine the shifts that build up cumulatively over the whole text as a result of the choices taken by the translator. Such analysis is carried out to understand the decision-making process underpinning the product of translation and infer the translator's textual-linguistic norms. Munday's approach is therefore descriptive, product- and process-oriented and data-driven. He derives his hypotheses from observing differences that occur in the parallel frequency 
lists and during the manual construction of the intercalated text. These initial hypotheses are then investigated with the aid of additional automatic methods of analysis such as aligned concordance lines. Munday's investigation of the first 800 words of his full-text parallel corpus reveals shifts in cohesion and word order that occur over the whole text and have the effect of moving the narrative viewpoint from the first to the third person and thereby distancing the reader from the thoughts, experiences and feelings of the main character in the story.

From the perspective of contrastive linguistics, Belinda Maia analyses the frequency and nature of the SVO sentence structure in English and Portuguese, particularly in those cases where the subject is realized by the first person pronoun I and $e u$, respectively, or by a name. The corpus she analyses is a small bidirectional parallel corpus comprising an English novel and its Portuguese translation and a Portuguese novel and its English translation. Maia considers this corpus design appropriate for comparing how the same situation is represented in the two languages in the parallel subcorpus, while the bilingual comparable subcorpus permits additional comparisons between the original languages and between the translational and the non-translational varieties of the same language. The discrepancies she observes in the frequencies of personal subjects (realized by either names or pronouns) suggest that, contrary to English language use, the seemingly subjectless $\mathrm{V}+\mathrm{O}$ sentence structure is the norm in original Portuguese, and translational Portuguese is influenced by English norms. Moreover, while the use of $I$ is syntactically necessary in English, the occurrence of the Portuguese equivalent $\mathrm{eu}$ seems to be related to pragmatic factors such thematisation, topicalisation and emphasis, while the verb acts as the normal theme of a high proportion of Portuguese sentences. On the basis of these results, Maia argues that the flexibility of word order and the wider variation of thematisation in Portuguese in relation to English allow for more subtlety in communication.

Like Maia, Jarle Ebeling regards parallel corpora as suitable sources of data for investigating the differences and similarities between languages, and adopts the notion of translation equivalence as a methodology for contrastive analysis. Ebeling uses the ENPC, a bidirectional parallel corpus of Norwegian and English texts, to examine presentative English 
there-constructions and the Norwegian equivalent det-constructions in original and translated English as well as original and translated Norwegian respectively. The corpus of original English reveals that be is by far the most frequent verb occurring in these structures, while Norwegian allows a much wider set of verbs, some of which in the passive voice. Ebeling's analysis of the Norwegian translation equivalents of the English there be-constructions reveals the influence of the target language. He finds that translators widen the range of det-constructions by using a) other verbs over and above those of existence, b) ha-existentials (corresponding to have-existentials in English), and c) det-constructions with passives. In Ebeling's view, this wider choice renders the meaning expressed in the translation more specific, that is more informative, compared with the original. Conversely, the English translations of det-constructions with lexical verbs in the active voice are very frequently rendered with there be-constructions, and this leads to less specification or 'despecification'. These results partly confirm the predictions put forward on the evidence provided by the analysis of the original corpora and throw new light on the assumed relationship of equivalence between these two structures in English and Norwegian.

With regard to the applied branch of corpus studies, the research carried out by Federico Zanettin and Lynne Bowker are of particular interest for translator training. Zanettin demonstrates how small bilingual comparable corpora are useful to explore the stylistic features of a particular text genre by comparing words and phrases that have a strong formal resemblance such as proper names and cognates or are based on lexicographic translation equivalents. Zanettin provides examples of such searches carried out in class with an Italian-English comparable corpus of leading daily newspapers. The way in which President François Mitterand is talked about in the two languages, for example, presents interesting differences: François Mitterand or simply Mitterand is commonly used in Italian, while English prefers President Mitterand or President François Mitterand or Mr Mitterand. Also, equivalent verbs typically used to introduce direct and reported speech have different frequencies as well as syntactic and collocational profiles in the two languages. Even cognates such as prezzi and prices show different collocational and colligational patterns. These data-driven learning 
investigations are valuable for refining contrastive knowledge of the source and target languages and enhancing translation skills.

Still within a pedagogic perspective, Bowker addresses two main problems usually faced by students training to become professional translators in specialized subject domains. One difficulty is shown by the occurrence of terminological errors resulting from poor subject-specific knowledge. The other is shown by the occurrence of errors due to a lack of specialized writing skills in the target language. Bowker's pilot study reports on a translation experiment undertaken with a group of fourth-year undergraduate L1 English students at Dublin City University who carried out two translations from L2 French of two semi-specialized passages on optical scanners. One translation was completed with the use of bilingual and monolingual dictionaries together with non-lexicographic reference materials (e.g. manuals and brochures). The other translation was carried out with a bilingual dictionary and a 1.4 million-word specialized monolingual corpus of English articles on optical scanners, which was compiled from Computer Select on CD-ROM. The software used to analyse the corpus was WordSmith Tools. The findings reveal that the corpus-aided translations were of higher quality in respect of subject field understanding (sensibilité aux nuances was accurately rendered as whatever their sensitivity to colour); correct term choice (vitrel glass paten or scan bed); and idiomatic expression (photodiodes sensibles à la lumière/light-sensitive photodiodes or photosensitive diodes). Bowker observes that, although there was no improvement with regard to grammar or register, the use of a specialized monolingual target corpus was not associated with poorer performance.

Revisiting the past, as we have done here, enables us to appreciate more than ever the value of this early research, which sowed the seed of the variegated lines of enquiry that, since the turn of the century, have contributed to the establishment, consolidation and growth of corpus studies of translation and interpreting in the pure and applied branches of the discipline, pushing the whole field of scholarship towards empiricism and interdisciplinarity. 


\section{CTIS: Twenty-five years on}

More than twenty-five years have passed since Baker (1993) put forward a research agenda for using corpora in descriptive translation studies and the first collection of papers on corpus-based translation and interpreting studies was published (Laviosa 1998). For the past two decades, Corpus-based Translation and Interpreting Studies (CTIS) has grown to such an extent that, already at the beginning of the 2000s, Baker $(2004,169)$ states that we "have too much rather than too little to go on."

Throughout these recent decades, many corpora have been compiled and extensively reviewed by the literature (e.g., Hu 2016; Laviosa 2002; Olohan 2004). Federico Zanettin (2012: 10) captures this overwhelming proliferation visually, as in Figure 1:

\begin{tabular}{|c|c|c|}
\hline $\begin{array}{c}\text { Comparable, monolingual } \\
\text { Originals + Translations } \\
\text { Language A + Language A }\end{array}$ & $\begin{array}{c}\text { Comparable, bilingual } \\
\text { Originals + Originals } \\
\text { Language A + Language B }\end{array}$ & $\begin{array}{c}\text { Parallel, bilingual } \\
\text { Original + Translation } \\
\text { Language A + Language B }\end{array}$ \\
\hline \multicolumn{2}{|c|}{ Reciprocal (bilingual, bidirectional, parallel) } \\
$\begin{array}{c}\text { Originals } \\
\text { Language A }\end{array}$ & $\begin{array}{l}\text { Translations } \\
\text { Language B }\end{array}$ \\
Translations & Originals \\
Language A & Language B \\
& & \\
\hline
\end{tabular}

Figure 1: Zanettin's (2012) typology of translation-related corpora.

And Figure 1 does not exhaust corpus mapping. Zanettin (2012: 11) himself complements it with other cases of corpus typologies:

A bilingual, reciprocal corpus may be graphically represented as a square cut across by diagonal lines, in which the different subcorpora stand at the corners. Multilingual, reciprocal parallel corpora may generate complex models described as a star and diamond configurations (Johansson 2003: 
139-142). In a star model, there are multiple translations of the same texts in different languages. The diamond model includes source texts in more than two languages as well as their translations in all the other languages.

With all these corpora, as Bernardini $\&$ Kenny (2020) confirm in a concise but thorough review, much of what was already underway in 1998 has been pursued further, especially with regard to the most prominent of all CTIS research paths - the study of the distinctive features of translational language (e.g. Othman 2020; Váradi 2007; Xiao 2010).

It is true that, with the turn of the millennium, early corpus-based studies have been complemented by newly-conceived alternative research lines, but, admittedly, to a comparatively much lesser extent. This is the case, for example, of explorations into translator style (e.g. Saldanha 2011). It is also the case of tentative incursions into multimodality, examining under-represented areas such as interpreting (e.g. Hu \& Tao 2014) or audiovisual translation (e.g. Baños et al. 2013). It is indeed the case of incipient approaches to translational data "produced under relatively new conditions" (Bernardini $\&$ Kenny 2020: 113), such as the work on (previously inexistent) modalities and genres like web localisation and social media translation (e.g. JiménezCrespo 2015). Nevertheless, as mentioned above, well into the 21st century, these alternative research routes are still in the periphery of CTIS.

The beginning of the 1990s, then, saw the official birth of CTIS thanks to a change in paradigm within TIS, made possible by the increasing strength of descriptive translation studies and the proliferation of ever more powerful electronic tools. In less than a decade, CTIS grew steadily (see Santamaría Urbieta \& Alcalde Peñalver, in this volume), and by the end of the 20th century, there was enough material for Laviosa (1998) to review the field for the first time. It seems to us that, in 2021, the time is particularly ripe for another, critical (self-)reflexive pause. We are not alone in this consideration.

De Sutter \& Lefer (2020), for example, put forward the same proposal in a particularly vehement manner when rallying for a new, updated research agenda for empirical translation studies, which they believe is "indispensable if we want findings in empirical translation studies to be accurate, reliable and generalisable so that we can start building solid, stable theories." (p. 7) Both authors (De Sutter \& Lefer 2020: 2) believe that this agenda would 
make it possible to advance in the study of largely unanswered questions relating to:

[the] mechanisms that shape translation, how these mechanisms interact, and to what extent this interaction functions differently than in other types of monolingual and bilingual written language production.

In their view, this new agenda, which is to be built upon particularly solid empirical pillars (and which is to be characterised by statistical sophistication, interdisciplinarity and a multi-methodological framework), is essential to overcome four main potentially lethal risks for CTIS:

(1) CTIS research is currently still focusing (to an excessive extent) on linguistic differences between translated and non-translated texts in order to identify distinctive translational features. Nevertheless, similarities are also an essential part of the picture to understand the process and product of translation (and interpreting), and this part of the picture, for De Sutter $\&$ Lefer (2020: 4), is mostly overlooked. In fact, for them:

with the benefit of hindsight, it is a questionable approach to assume first and foremost differences when translated texts in a given language that are produced by highly skilled, native-language professionals are compared with texts in the same language produced by presumably equally skilled language professionals (journalists, writers, spokesmen, etc.), with the only obvious difference being the circumstances under which the texts are produced (bilingual vs monolingual language activation). [...] In other words, subtle quantitative differences are likely to be found across translated and non-translated texts, alongside a massive number of commonalities.

(2) There is much room for improving the CTIS theoretical framework, which both scholars (De Stutter \& Lefer 2020: 4-5) perceive as "under-developed." The reason for this is that:

Instead of empirical research in translation studies giving rise to the falsification, verification or adaptation of the hypotheses of universal features of translation, as initially intended by Baker, universal features have been used repeatedly and uncritically to 'explain' specific patterns observed in the corpus data. In the process, translation universals have gradually lost power in that they have only been used as fixed, passe-partout post hoc explanations: whatever linguistic phenomenon is being studied, there 
will always be some translation universal available which can be used to rationalise the descriptive patterns uncovered in the data.

(3) There is a lack of sophistication (i.e. a basic, often incorrect, use of statistics) in CTIS research designs, which end up nurturing reductionist (monofactorial) studies. The result of this is that many CTIS studies often rely on a single explanatory factor (notably the distinction between translated and non-translated texts) to characterise the process and product of translation (and interpreting). De Sutter \& Lefer (2020: 5) quote Gries (2018: 295) to boldly declare that:

monofactorial observational studies have virtually nothing to contribute to corpus linguistics [because] (i) no phenomenon is monofactorial and (ii) even if one had a new monofactorial hypothesis of a phenomenon, it would still require multifactorial testing to determine (a) whether it either adds anything to what we already know about the phenomenon (by statistically controlling for what we already know) or (b) whether it replaces (parts of) what we already know about the phenomenon.

(4) CTIS remain largely isolated and fixed on their object of study: translated/ interpreted products and process

without taking into account theoretical and methodological developments in other, related, fields such as corpus linguistics (including learner corpus research), variational linguistics, contrastive linguistics, sociolinguistics, psycholinguistics and cognitive linguistics, to name but a few (2020: 5).

In the authors' views, their proposed research agenda is not totally new in the field. It is already being upheld by works such as Delaere \& De Sutter (2013), Hu et al. (2019) and Kruger (2019), to name but a few. Furthermore, it is disseminating quickly with the following declaration of intentions:

understanding of the governing principles underlying translation, and the constraints under which it operates, can be achieved, in our view, by re-adopting and updating the essential aspect of Baker's research program, i.e., looking over the disciplinary fence and carefully selecting corpus-linguistic, ethnographic, sociological, and psycholinguistic methods that are appropriate for studying central aspects of translation, as well as interpreting research outcomes in an emerging, bottom-up translation theory that builds on theories in neighboring disciplines, such as contact linguistics, second language acquisition research and psycholinguistics (2020: 2). 
Like De Sutter \& Lefer, we believe that another revamping impulse to Baker (1993)'s original agenda seems more than fitting with the turn of yet another decade, where ravishingly new texts are being created, and new contexts are being populated. It seems to us this the only way we can continue to follow Tymoczko's (1998: 653) advice "to move from text-based questions to context-based questions."

Nevertheless, we are more cautious in our evaluation of risks. It is true that differences have been the crux of the matter in the field for some time now and that similarities are undoubtedly an under-researched part of the translational picture. It is, however, intriguing to see how, in certain quarters, the debate seems to be incipiently shifting from translation and interpreting (as the objects of study of independent disciplines) to a somewhat larger space populated by "similar" subgenres, those of constrained communication, within which (C)TIS might be losing the limelight that has been so difficult to occupy. While it is true that translation theory is enhanced by high quality empirical research, it is also true that theoretical advances cannot and will not just come from empiricist minds. Disregarding non-empiricist approaches to translation and interpreting (like those coming from so many valuable corners within (C)TIS) would be disowning our (C)TIS history. We would indeed do well to pursue more sophistication (in our hopefully increasingly refined statistics-based approaches). Nonetheless, it seems to be equally true that the complex phenomena of translation and interpreting cannot be fully grasped by these or those metrics only and that our past has already warned us against the false illusion of indisputable truth (Venuti 2000). Finally, when has (C)TIS ever been isolated? If there is one quality in our studies, this is that we have never ceased to "look over the disciplinary fence." We have been continuously inspired by literary criticism; informed by linguistic analysis; empowered by (post-modern) cultural, postcolonial, queer, gay studies; nurtured by psycholinguistic methods; complemented by sociological approaches, and so forth.

In fact, it is by looking yet once again over the disciplinary fence that we realise CTIS is not the only field where pausing and self-reflexivity is being called for. Neighbouring fields, such as Corpus-Assisted Discourse Analysis (CADS), are addressing very similar needs, as the brilliant monograph published by Taylor \& Marchi (2018) testifies. This is not surprising 
in the least. CTIS and CADS share similar goals when "us[ing] corpora to study how social realities are constructed, represented and transmitted linguistically" (2018: 1) (through monolingual or multilingual artefacts). They both share similar foundations (some of which can be found in corpus linguistics and discourse analysis). They have both grown exponentially over the past decade, with the emergence/reinforcement of conference series (e.g. the corpora and discourse series in CADS; the CILC conferences in CTIS) and the increased prominence of well-known publications. And when Taylor $\&$ Marchi (2018: 2) point at areas of concern for CADS (i.e. lack of standardisation, hindrance of institutional barriers, concerns about decontextualised data, epistemological issues...), they ring a loud bell within CTIS circles. It is then hardly surprising that, after the astounding success of recent years, both CTIS (with the leadership of De Sutter $\&$ Lefer, among others) and CADS (with Taylor \& Marchi inter alios) are converging into the very same conclusion: "The time has come to pause and reflect on what it is we do." In Taylor \& Marchi's (2018: 2) words:

This is not intended as an assault of the exciting work which is emerging but a recognition of the maturity of the methodology, which is now robust enough to withstand, and indeed demand, close scrutiny.

According to Taylor \& Marchi (2018), close scrutiny (i.e. a self-reflexive form of awareness) is intended to fight three inextricably connected potential types of malaise: partiality, dusty corners and blind spots. In other words, focusing on certain areas while disregarding others brings about an incompleteness (i.e. a partiality) in research that leaves a series of dusty corners or overlooked features (such as similarities or absence) and under-researched contents (minoritised topics, text-types and languages that are hidden by dominant voices). It also creates black holes of undetected or under-analysed components (blind spots) that can only be illuminated with innovative approaches, including those inspired by triangulation. If we think about it, this argument overlaps with what is advocated by De Sutter $\&$ Lefer. An (excessive?) concentration on the distinctive translational features leaves behind dusty corners with overlooked features (such as similarities) and under-researched contents (e.g. many of the mechanisms that shape 
translation) and black holes in translational theory than can only be explored with higher levels of complex and sophisticated multi-methods.

In line with De Sutter \& Lefer (2020) and Taylor \& Marchi (2018) among others, this volume is born with the intention to encourage CTIS practitioners to pause and look around; to explore dusty corners and blind spots; to fight partiality, while injecting doses of innovation into our work. It is ultimately intended to boost critical thinking, (self-)awareness and (self)reflexivity, without renouncing to our past. Quite the contrary, while embracing what we conceive of as a glorious past. Always looking outwards (over the fence), always gazing forward (while peeping into the rear mirror). In this spirit, the volume is organised in such a way that it covers four main themes: Translation Features as a Starting Point; Neglected and Overlooked Areas of Study; Researching Original and Translated Communication Produced under New Conditions and Self-Reflexivity.

We set off the volume by tightening our unbreakable bondage with the past when taking up CTIS' most prolific research concern: translational features. In a descriptive-explanatory proposal ("Explicitation and Implication in Translation: Combining Comparable and Parallel Corpus Methodologies"), Miguel Ángel Jiménez-Crespo \& Maribel Tercedor-Sánchez concentrate on one of the most widely researched of these features, explicitation. They do so through both comparable and parallel corpora. Along these lines, not only do they identify cases of explicitation (and implicitation) regarding the under-researched issue of Latin-Greek terms (LG) in medical texts, but they also delve into the potential causes behind this explicitation (i.e. cross-linguistic interference, risk-aversion...). This approach is in line with other recent empirical works on the topic (see, for example, Delaere \& De Sutter 2013; Kruger 2019). In a nutshell, Jiménez-Crespo \& Tercedor-Sánchez present us with the unique and complex case of LGs, where inter and intra-linguist translation processes converge, and where explicitation is intertwined with determinologisation.

The next four contributions revolve around the theme of what we could call Neglected and overlooked areas of study. Now we enter realms that are not frequently visited by corpus-based translation and interpreting scholars, such as subtitling, travel journalism, simultaneous interpreting and operatic audio description. Stepping on the (partially) untrodden is gradually 
taking on in CTIS. When entering these realms, all four articles look over the disciplinary fence (some do so on more than one occasion) in order to find adequate (multi)-methods with a view to analysing their neglected and overlooked objects of study.

Blanca Arias' "Using Corpus Pattern Analysis for the Study of Audiovisual Translation. A Case Study to Illustrate Advantages and Limitations", for example, turns to the Theory of Norms and Exploitations (from Television Studies) and the application of Corpus Pattern Analysis (CPA) (from lexical studies) in order to perform an analysis into creative (anomalous or non-canonical) collocations. The corpus chosen for her exploration consists of original English and translated Spanish subtitles from the first two episodes of the first season of television series Castle (2009), Dexter (2006) and The Mentalist (2008). Studies of creativity (or exploitations in Arias' terminology) such as this are certainly under-represented. They are particularly welcome when they conclude with the critical assessment of the advantages and disadvantages of importing (CPA) methodology.

Collocations are also the topic of the article by David Finbar Brett, Barbara Loranc, Antonio Pinna ("A Corpus-Driven Analysis of Adjective+Noun Collocations in Travel Journalism in English, Italian and Polish"), in which travel journalism is explored through the corpus-driven lens. With the aid of three comparable corpora of travel reportage in English, Italian and Polish, the authors explore a variety of issues: (a) similarities and differences in the frequencies of adjective/noun collocations across languages; (b) similarities and in the denotative effects of some of the most frequent adjective/ noun collocations; (c) syntactic variability of the collocates; (d) connectivity of some of the most productive collocates; and (e) collocational behaviour within selected themes. Among the paper's innovations, the cross-linguistic exploration of collocations in travel writing (certainly an under-researched topic of analysis) immediately springs to mind. Nevertheless, there are other sources of methodological innovation. Firstly, imported tools from the Social Network Analysis (such as Gephi) are used to reinforce the notion of collocational connectivity (which is built upon the concept of relational networks). Secondly, automatised protocols of compilation and annotation (with tailor-made Perl scripts created by the authors themselves) are a necessary step towards those higher levels of (programming) sophistication requested 
by De Sutter \& Lefer (2020) for their new agenda. We believe greater programming autonomy by CTIS researchers is an increasingly unavoidable requirement in the field.

Collocation and methodological sophistication are yet again the main ingredients of Marta Kajzer-Wietrzny \& Łukasz Grabowski's "Formulaicity in Constrained Communication: An Intermodal Approach." Here collocation revolves around the notion of the bigram. For its part, sophistication is of a different type to that of the previous contribution and results from proficient knowledge of statistics. Mastering increasingly complex statistics (like being autonomous in programming, as shown in the previous article) is also becoming an unavoidable requirement for present and future CTIS research. In short, this article fits a Poisson regression model with fixed and random effects to dissect the use of bigrams in subcorpora from the intermodal EPTIC corpus. The result is a comparative study of three types of parliamentary speeches in English: translated speeches from Polish originals; interpreted speeches from Polish originals and original speeches produced by Polish natives for whom English is a second language. The neglected area of simultaneous interpreting and the increasingly widespread interest in constrained communication are particularly suitable for the present volume. The multidimensional nature of the study is also worth noting. In De Sutter \& Lefer's (2020) manner, this article goes beyond the monofactorial explanation of the communicative phenomena under analysis to assess causation along five dimensions: (1) (mono-bilingual) language activation; (2) (spoken, written-multimodal) modality and register; (3) (unmediated/mediated) text production; (4) (native, non-native) proficiency; and (5) (high/low) task expertise. Finally, the paper resorts to a set of under-used tools within CTIS (such as Formulib, R or ad-hoc scripts in Python) to complement our basic toolkit.

"The Hierarchization of the Operatic Signs through the Lens of Audio Description: A Corpus Study" by Irene Hermosa-Ramírez also deals with a variety of very dusty corners that certainly benefit from the exploration with corpus-based tools. CTIS has not often been to the opera, nor have we indulged in examining operatic audio description. Spotting similarities and differences between productions from two famous opera houses (the Teatro Real, in Madrid, and the Liceu, in Barcelona) has not been a common aim 
for the field. Complementary semiotic analyses do not usually come hand in hand with well-established corpus-based instruments. All this dusting is precisely what Hermosa-Ramírez does in her article. She compiles two corpora with audio descriptions of three famous operatic productions (Aida, The Magic Flute and Carmen) as represented at the Teatro Real and the Liceu and, with the aid of Sketch Engine, she compares mean sentence length, open class word frequencies, POS distributions and TTRs. Having done this, she complements her results with a semiotic analysis based on Rędzioch-Korkuz (2016) and TRACCE narratology tagset. Furthermore, she shows that triangulation is not the only possibility when using mixed-method designs. Methodological complementation is a powerful way to fight partiality.

The theme of Researching Original and Translated Communication Produced under New Conditions strongly links the next two articles, dealing with topics that were mostly unheard of in the 1990s.

In "Los estudios de corpus y la localización: una propuesta de análisis para material interactivo / Corpus studies and localization: a research proposal for interactive material", by Laura Mejías Climent, English original and Spanish translated dubbing synchronies of three video games (Batman: Arkham Knight, Assassin's Creed Syndicate and Rise of the Tomb Raider) are the main focus of the study. This topic in itself is already pretty innovative. Triangulation of data from qualitative methods, quantitative results and professional knowledge drawn from semi-structured interviews adds complexity to the standpoints from which the study is performed. The theoretical inspiration from localisation, audiovisual translation and corpus-based studies generates synergies that result in some sophisticated components. For example, multimodality here incorporates not just audiovisual stimuli but also tactile inputs. Moreover, the unit of analysis distances itself from the most traditional understanding of original and translated text and concentrates on "game situations." This generates the most innovative aspect of this article's already innovative proposal. Original and translated texts do not pre-date this research but are both a result and a means of it. In order for the researcher to research, she has to play the games and compare her moves. Setting out to study inexistent text was not a common goal during the 1990s. However, if we come to think about it, it is particularly characteristic of our 
current "liquid" times (Bauman 2000), where the materiality of virtual/real, existent/inexistent texts is difficult to grasp.

Leticia Moreno-Pérez \& Belén López-Arroyo, for their part, have an equally updated focus (i.e., a writing generator and its use for the translating profession) in "A Typical Corpus-Based Tool to the Rescue: How a Writing Generator Can Help Translators Adapt to the Demand of the Market". As is well known, nowadays a writing generator is a tool that helps non-native speakers to generate text (within specialised genres) in a foreign language. Developing a writing generator is an undoubtedly sophisticated task that enriches our current CTIS toolkit. It relies on file managers, tagger builders and taggers that produce information on prototypical rhetorical structures (moves and steps), lexicon-grammatical patterns and terminological and phraseological glossaries. Its application to translation within the field of oenology shows CTIS is not only able to look over the disciplinary fence but also (and mainly) to listen to the demands of the market, narrowing the breach between academia and the profession.

Last but certainly not least, the volume concludes with Self-Reflexivity, the ultimate goal of this venture. Self-reflexivity can be of two types. The first type is that of individual self-reflexivity, in which researchers analyse their prior/contemporary production to reinforce their theoretical and methodological standing. The second type of self-reflexivity is collective and disciplinary-bound; it looks over the field fence while focusing on the domain kept within that fence.

"Autocrítica de publicaciones previas basadas en corpus: análisis DAFO / Self-Criticism of Previous Corpus-Based Publications: SWOT Analysis", by Alexandra Santamaría Urbieta \& Elena Alcalde Peñalver, is an example of the first type of self-reflexivity. The authors review four of their own joint publications in which corpus-based methods are applied. For this purpose, they resort to the SWOT (Strengths, Weaknesses, Opportunities and Threats) methodology, which is particularly prolific in businesses and marketing studies and which provides a useful scaffolding for self-criticism and self-awareness. We believe this is a good way for CTIS partitioners to contribute to the revamping of Baker's (1993) agenda.

And it is precisely Mona Baker who is a powerful inspiring voice in the concluding contribution to the volume, "From Text to Data: Mediality in 
Corpus-based Translation Studies", by Jan Buts \& Henry Jones. This final article is produced within the Genealogies of Knowledge (GoK) project framework, where Baker (a Professor Emeritus at present) has a prominent role. Indeed, Buts' \& Jones' proposal is an example of the second type of self-reflexivity, whereby scholars dare to ponder on fundamental pillars of the discipline precisely as the only manner to make it stronger. One of such pillars within CTIS is the electronic media "in and through which translations are stored, transmitted and - by extension - studied (Armstrong 2020; Pérez-González 2014)" (see contribution in this volume). These media are not mere containers that serve to preserve and convey meaning and knowledge in an untainted manner. On the contrary, they are transformative environments that deeply affect our relation with (and understanding of) texts. On the whole, CTIS seems to have pushed aside issues of mediality, which have been confined to some of our dustiest corners. Yes, as Buts \& Jones argue, the limitations and restrictions of technologies have been discussed before. However, as the authors advocate: "[w] hatever the cause, the convertibility of the sign and its attachment to the binary standard are yet to be consistently questioned." Being aware that the electronic media upon which CTIS depends and the software tools with which CTIS performs its analyses lead to the application of certain (pattern-seeking) methodologies rather than others (more focused on structures and narratives) is the first step to combatting partiality. Designing new visualisation tools and critically applying them to the examination of political and scientific concepts - as Buts $\&$ Jones do as part within the GoK project — seems to us a powerful initiative to contribute to CTIS new agenda.

In the dawn of the second decade of the 21st century, when in many parts of the world citizens in lockdowns hold their breath without a clear idea of what to do next, a need to stop and think critically is indeed more pressing than ever. 


\section{References}

BAKER, Mona. (1993) "Corpus Linguistics and Translation Studies — Implications and Applications." In: Baker, Mona; Gill Francis \& Elena Tognini-Bonelli (eds.) 1993. Text and Technology. Amsterdam: John Benjamins, pp. 233-50. BAKER, Mona. (1998) "Réexplorer la langue de la traduction: une approche par corpus.” In: Laviosa, Sara (ed.) 1998. L'Approche Basée sur le Corpus/The Corpus-based Approach. Special Issue of Meta 43:4, pp. 480-485.

BAKER, Mona. (2004) "A Corpus-Based View of Similarity and Difference in Translation." International Journal of Corpus Linguistics 9:2, pp.167-93.

BAÑOS, Rocío; Silvia BRUTI \& Serenella ZANOTTI. (2013) "Corpus Linguistics and Audiovisual Translation: In Search of an Integrated Approach." Perspectives 21:4, pp. 483-90.

Bauman, Zygmunt. (2000) Liquid Modernity. Cambridge: Polity Press.

Bernardini, Silvia \& Dorothy Kenny. (2020) "Corpora." In: Baker, Mona \& Gabriela Saldanha (eds.) 2020. Routledge Encyclopedia of Translation Studies. Third edition. London \& New York: Routledge, pp. 112-15.

BOWKER, Lynne. (1998) "Using Specialized Monolingual Native-Language Corpora as a Translation Resource: A Pilot Study.” In: Laviosa, Sara (ed.) 1998. L'Approche Basée sur le Corpus/The Corpus-based Approach. Special Issue of Meta 43:4, pp. 631-651.

De SutTer, Gert \& Marie-Aude Lefer. (2020) "On the Need for a New Research Agenda for Corpus-Based Translation Studies: A Multi-Methodological, Multifactorial and Interdisciplinary Approach." Perspectives 28:1, pp. 1-23. Delaere, Isabelle \& Gert De Sutter. (2013) "Applying a Multidimensional, Register-Sensitive Approach to Visualize Normalization in Translated and Non-Translated Dutch." Belgian Journal of Linguistics 27, pp. 43-60.

Ebeling, Jarle. (1998) "Contrastive Linguistics, Translation, and Parallel Corpora.” In: Laviosa, Sara (ed.) 1998. L’Approche Basée sur le Corpus/The Corpus-based Approach. Special Issue of Meta 43:4, pp. 602-615.

HALVERSON, Sandra. (1998) "Translation Studies and Representative Corpora: Establishing Links between Translation Corpora, Theoretical/Descriptive Categories and a Conception of the Object of Study." In: Laviosa, Sara (ed.) 1998. L'Approche Basée sur le Corpus/The Corpus-based Approach. Special Issue of Meta 43:4, pp. 494-514.

Hu, Kaibao. (2016) Introducing Corpus-Based Translation Studies. New Frontiers in Translation Studies. Berlin \& Heidelberg: Springer Berlin Heidelberg. 
HU, Kaibao \& Qing TAO. (2014) "The Chinese-English Conference Interpreting Corpus: Uses and Limitations." Meta 58:3, pp. 626-42.

Hu, Xianyao; Richard XiaO \& Andrew Hardie. (2019) "How Do English Translations Differ from Non-Translated English Writings? A Multi-Feature Statistical Model for Linguistic Variation Analysis." Corpus Linguistics and Linguistic Theory 15:2, pp. 347-82.

JiMÉNEZ-CRESPO, Miguel A. (2015) "Translation Quality, Use and Dissemination in an Internet Era: Using Single-Translation and Multi-Translation Parallel Corpora to Research Translation Quality on the Web." The Journal of Specialised Translation 23, pp. 39-63.

KRUGER, Haidee. (2019) "That Again: A Multivariate Analysis of the Factors Conditioning Syntactic Explicitness in Translated English." Across Languages and Cultures 20:1, pp. 1-33.

LAviosa, Sara. (1998a) "The English Comparable Corpus: A Resource and a Methodology." In: Bowker, Lynne; Michael Cronin; Dorothy Kenny \& Jennifer Pearson (eds.) 1998. Unity in Diversity? Current Trends in Translation Studies. Manchester: St. Jerome, pp. 101-112.

Laviosa SARA. (1998B) "Core PATterns of LeXical Use in a Comparable Corpus of

ENGLiSH NARRATIVE PROSE." IN: LAVIOSA, Sara (ed.) 1998. L’Approche Basée sur le Corpus/The Corpus-based Approach. Special Issue of Meta 43:4, pp. 557-570. LAVIOSA, Sara (ed.) (1998) Special Issue: L'approche Basée Sur Le corpus/The Corpus Based Approach. Meta. Journal Des Traducteurs/Meta. Translators' Journal 43:4. LaviosA, Sara. (2002) Corpus-Based Translation Studies: Theory, Findings, Applications. Amsterdam: Rodopi.

MAIA, Belinda. (1998) "Word Order and the First Person Singular in Portuguese and English.” In: Laviosa, Sara (ed.) 1998. L’Approche Basée sur le Corpus/The Corpus-based Approach. Special Issue of Meta 43:4, pp. 589-601.

MALMKJÆR, Kirsten. (1998) "Love thy Neighbour: Will Parallel Corpora Endear Linguistics to Translators?" In: Laviosa, Sara (ed.) 1998. L'Approche Basée sur le Corpus/The Corpus-based Approach. Special Issue of Meta 43:4, pp. 534-541. MundaY, Jeremy. (1998) "A Computer-Assisted Approach to the Analysis of Translation Shifts.” In: Laviosa, Sara (ed.) 1998. L’Approche Basée sur le Corpus/The Corpus-based Approach. Special Issue of Meta 43:4, pp. 542-556. Olohan, Maeve. (2004) Introducing Corpora in Translation Studies. London \& New York: Routledge. 
Othman, Waleed. (2020) "An SFL-based model for investigating explicitation-related phenomena in translation." In: Calzada Pérez, María \& Jeremy Munday (eds.) Meta: Journal des traducteurs 65:1, pp. 193-210.

ØvERÅs, Linn. (1998) "In Search of the Third Code: An Investigation of Norms in Literary Translation." In: Laviosa, Sara (ed.) 1998. LAApproche Basée sur le Corpus/The Corpus-based Approach. Special Issue of Meta 43:4, pp. 571-588. RęDzIOCH-KORKUZ, Anna. (2016) Opera Surtitling as a Special Case of Audiovisual Translation. Bern: Peter Lang.

SALDANHA, Gabriela. (2011) "Translator Style: Methodological Considerations." The Translator 17:1, pp. 25-50.

SHLESINGER, Miriam. (1998) "Corpus-based Interpreting Studies as an Offshoot of Corpus-based Translation Studies.” In: Laviosa, Sara (ed.) 1998. L’Approche Basée sur le Corpus/The Corpus-based Approach. Special Issue of Meta 43:4, pp. 486-493.

TAYLOR, Charlotte \& Anna MARCHI (eds.) (2018) Corpus Approaches to Discourse: A Critical Review. Milton Park, Abingdon, Oxon \& New York: Routledge.

TyMOCZKO, Maria. (1998) "Computerized Corpora and the Future of Translation Studies." Meta 43:4, pp. 652-60.

VÁRADI, Tamas. (2007) "NP Modification Structures in Parallel Corpora." In: Rogers, Margaret \& Gunilla Anderman (eds.) 2007. Incorporating Corpora. The Linguist and the Translator. Clevedon: Multilingual Matters.

Venuti, Lawrence. (2000) “SSerá Útil La Teoría de La Traducción Para Los Traductores?" Vasos Comunicantes 16, pp. 26-34.

XIAO, Richard. (2010) "How Different Is Translated Chinese from Native Chinese?: A Corpus-Based Study of Translation Universals." International Journal of Corpus Linguistics 15:1, pp. 5-35.

ZANETTIN, Federico. (1998) "Bilingual Comparable Corpora and the Training of Translators." In: Laviosa, Sara (ed.) 1998. L'Approche Basée sur le Corpus/The Corpus-based Approach. Special Issue of Meta 43:4, pp. 616-630.

Zanettin, Federico. (2012) Translation-Driven Corpora Corpus Resources for Descriptive and Applied Translation Studies. Translation Practices Explained. Manchester \& Kinderhook: St. Jerome Pub. 


\section{BIONOTE / NOTA BIOGRÁFICA}

María Calzada Pérez is Full Professor of Translation Studies at the Universitat Jaume I. (Spain). Her research mainly focuses on corpus-based translation studies, institutional translation (especially at the European Parliament), ideology, and translator-teaching. She is Coordinator of the ECPC (European Comparable and Parallel Corpora of Parliamentary Speeches) research group. She has produced research, such as (i) Transitivity in Translating: The Interdependence of Texture and Context (Peter Lang, 2007); (ii) "Five Turns of the Screw. A CADS analysis of the European Parliament" (Journal of Language and Politics 16:3, 2017) (iii). "Corpus-based methods for Comparative Translation and Interpreting Studies" (Translation and Interpreting Studies 12:2, 2017); and (iv) "What is kept and what is lost without translation? A corpus-assisted discourse study of the European Parliament's original and translated English" (Perspectives 26:2, 2017). She is also editor of volumes like: Apropos of Ideology (St. Jerome, 2003).

SARA LAVIOSA is Associate Professor in English Language and Translation Studies at the Department of Lettere Lingue Arti Italianistica e Culture Comparate (LeLiA), Università degli Studi Aldo Moro, Italy. Her research interests are in corpus-based translation studies and pedagogic translation. She is the author of Corpus-based Translation Studies (Rodopi/Brill, 2002), Translation and Language Education (Routledge, 2014) and Linking Wor(l)ds (Liguori, 2020). She is the co-author (with Adriana Pagano, Hannu Kemppanen \& Meng Ji) of Textual and Contextual Analysis in Empirical Translation Studies (Springer, 2017). She is the guest editor of LApproche Basée sur le Corpus/The Corpus-based Approach (Special Issue of Meta 43:4, 1998), Translation in the Language Classroom (Special Issue of The Interpreter and Translator Trainer 8:1, 2014). She is the co-editor (with Maria GonzálezDavies) of The Routledge Handbook of Translation and Education (Routledge, 2020) and (with Meng Ji) of The Oxford Handbook of Translation and Social Practices (OUP, 2020). She is the Founder and Editor of the journal Translation and Translanguaging in Multilingual Contexts (John Benjamins). 